\title{
Concept identification as a function of relevance of pretraining and percentage of informative feedback
}

GEDIMINAS NAMIKAS

WESTERN WASHINGTON STATE COLLEGE

Concept identification performance as a function of 3 types of verbal pretraining was compared under complete (100\%) informative feedback (IF) with performance under reduced IF (75\%, 50\%, and 25\%). The verbal pretraining was either relevant (R), irrelevant (IR) or neutral (C) with respect to the dimensions involved in the concept identification task. It was concluded that (a) $\mathrm{R}$ pretraining facilitates concept identification performance, and (b) a reduction in IF to $50 \%$ or less degrades concept identification performance.

Gelfand (1958) and Namikas (1962) demonstrated that pretraining with verbal labels for relevant stimulus attributes or dimensions facilitates subsequent concept identification. Pretraining with labels for irrelevant stimulus attributes, however, did not produce a corresponding degradation of concept identification behavior. The present study was designed to investigate the possibility that the informative feedback (IF) conditions that were used in the two experiments may have accounted for the lack of effect of irrelevant (IR) pretraining.

Any incorrect hypotheses or response sets due to IR pretraining could have been rapidly disconfirmed, since, in these studies, IF was provided after every response $(100 \%$ IF). A reduction of IF to something less than this level, as in the present experiment, was expected to make such a disconfirmation more difficult and lead to a decrement in concept identification performance after IR pretraining. In addition, the effect of reduced IF on ease of concept identification itself was investigated, using a wider range of reduced IF conditions than had been reported by previous investigators (Bourne \& Pendleton, 1958).

Experiment 1.

Ninety students from introductory psychology courses were assigned, in order of their appearance, to one of 18 groups. The $\mathrm{S}$ sat facing a black wooden panel (19 in. high $\times 35$ in. wide). In the center of the panel there was an aperture ( 3 in. high $x 5$ in. wide) which was closed by a shutter between stimulus presentations. Four evenly spaced lights were mounted on the panel directly below the opening to provide IF to the $\mathrm{S}$. The response apparatus consisted of four pushbuttons which were mounted on a black wooden box ( 3 in. high $x 4-1 / 2$ in. wide $x 14$ in. long) placed directly in front of the $S$.

On E's side of the panel, four lights indicated the responses made by the $S$. A rotary switch, preset by the $\mathrm{E}$ for each stimulus, controlled the IF lights. A deck of stimulus cards was positioned behind the opening in the panel. When the $E$ raised the shutter, the $S$ was able to see the first card. The next stimulus was exposed automatically when the first card was removed.

The pretraining task was presented under the guise of a reaction time experiment. The $S$ responded to each of four words 10 times. Each word had been typed in the center of a $3 \times 3 \mathrm{in}$. card. Two of these sets of words referred to either relevant (R) or irrelevant (IR) attributes of the transfer stimuli. The third set consisted of four neutral words and was used for the control (C) pretraining condition. Each $\mathrm{S}$ was shown the four words and was told that two of the words (e.g., "red" and "ellipse") belonged in one category, while the other two words (e.g., "green" and "circle") were to be placed in a second category. Only two response buttons were available during the pretraining task, each button representing one category. Verbal feedback was provided after each categorization response.

The words were presented in a random sequence which was constructed with one restriction, namely, that a word would not follow itself. One such random sequence was constructed for each replication.

Immediately after the completion of the pretraining session, the $\mathrm{E}$ inserted the two additional response buttons in the response apparatus and instructed the $S$ that he would have to perform the same type of task with another series of stimuli each of which had to be categorized into one of four categories. The function of the feedback lights was explained and those Ss who were to work under reduced IF conditions were told that IF sometimes would not he given. The $\mathbf{E}$ stressed that lack of IF indicated neither that the response was correct nor that it was incorrect.

The transfer stimuli varied in six bi-level dimensions: form (circle or ellipse), color of figure (red or green), size of figure (large or small), number of borders (one or two), type of border (solid or broken), and color of border (blue or yellow). ${ }^{2}$ Either the form and color of the figure or the type and number of borders was relevant for a given problem. The size of the figure and the color of the border were always irrelevant. All possible combinations of the attributes were used to produce a deck of 64 cards $\left(2^{6}=64\right)$.

The stimuli were presented in a different random sequence for each replication. Two restrictions were used in constructing the sequences: (a) no two examples of the same category were presented successively, and (b) four examples of each of the four categories were presented in the first block of 16 stimuli and in every successive block of 16 stimuli. 
In addition, a different random sequence was constructed for blank (no IF) trials for each replication. Two restrictions were also applied in this case: (a) no more than four successive blank trials were given anywhere in the sequence, and (b) the proportion of blank trials in successive blocks of 16 stimuli was the same for a given percentage of reduced IF.

The task was self paced and no correction was allowed. Each categorization response was followed by the presentation of the correct feedback light, except on blank trials, and the shutter was lowered until the presentation of the next stimulus. The criterion for the concept identification task was either 32 correct consecutive responses or two complete presentations of the stimulus deck (128 trials).

The experimental design was a 3 by 2 by 3 orthogonal design. The Ss received either $R$, IR, or C pretraining. The same pretraining condition represented either $\mathbf{R}$ or IR pretraining depending upon which of two problems was used as the transfer task. Finally, IF was provided on either 100,75 , or $50 \%$ of the trials on the concept identification task. Five Ss were run in each group for a total of $90 \mathrm{Ss}$.

Experiment 2.

The results of the flrst study indicated that a reduction of IF to the $50 \%$ level did not represent a limiting condition as far as concept identification was concerned. The investigation of the effects of reduced IF was extended, therefore, to a lower percentage of IF (25\%). The Ss again received either $R$, IR, or $C$ pretraining and transferred to one of two problems. Five Ss were run in each of the six groups thus formed $(\mathrm{N}=30)$. These were also drawn from introductory psychology courses.

\section{Results and Discussion}

While a total of $36 \mathrm{Ss}$ did not reach the criterion of 32 correct consecutive responses by the end of 128 trials, only five of these fallures occurred after $R$ pretraining. The rest were almost evenly divided between the C and IR groups. A Chi-square test indicated that $\mathbf{R}$ pretraining led to a disproportionally smaller number of fallures (Chi-square $=6.167, \mathrm{df}=2, .05>\mathrm{p}>.02$ ). When the Ss who were able to verbalize the concept correctly at the end of the experimental session were eliminated from these data, however, the differences in the number of failures as a function of type of pretraining were found to be negligible. This suggests that after $R$ pretraining fewer trials are necessary for correct concept identification and the $S$, therefore, is more likely to reach the criterion of 32 correct consecutive responses within the limit of 128 trials.

A comparison of the number of fallures as a function of the percentage of IF indicated that the differences were not signiflcant, even though there was an apparent trend for a greater number of failures to occur with a decrease in IF (Chi-square $=6.00, d f=3, .20>p>.10$ ).

An analysis of variance on the errors-to-criterion ${ }^{3}$ data showed that the two problems were of equal difficulty; however, both main effects of type of pretraining and percentage of IF proved to be highly significant ( $F$ $=8.25, \mathrm{df}=2 / 108, \mathrm{p}<.001$, and $F=12.11, \mathrm{df}=3 / 108, \mathrm{p}<$ .001 , respectively). A Multiple-Range test (Duncan, 1955) indicated that fewer errors were made by Ss after $\mathrm{R}$ pretraining than either after $\mathrm{C}$ or IR pretraining (1\% level of confidence). The latter groups were not significantly different from each other (mean errors were: $\mathbf{R}$ $=23.6, \mathrm{C}=39.2, \mathrm{IR}=43.9$ ). A comparison of mean errors as a function of the percentage of IF showed that the $100 \%$ and $75 \%$ IF groups made significantly fewer errors than the $50 \%$ or $25 \%$ IF groups (1\% level of confidence). The reduction in IF from $100 \%$ to $75 \%$ or from $50 \%$ to $25 \%$ did not produce a significant increase in errors (mean errors were: $100 \%$ IF $=23.3,75 \%$ IF $=23.1,50 \%$ $I F=42.5$, and $25 \%$ IF $=53.2$ ).

These results support the conclusion that $\mathbf{R}$ pretraining facilitates concept identification performance. The facilitative effect is independent of the percentage of IF, at least within the range of percentages investigated in the present study. The consequences of IR pretraining are not significantly different from the consequences of $\mathrm{C}$ pretraining, irrespective of the percentage of IF. Both of these conclusions are in agreement with the results obtained in previous investigations (Gelfand, 1958; Namikas, 1962).

A reduction in percentage of IF degrades concept identification performance. Feedback has to be reduced substantlally (to less than 75\% IF in this case) before the effect is observable, however. These conclusions are in general agreement with the results of a study by Bourne \& Pendleton (1958). These investigators reported, however, that reductions in feedback smaller in magnitude than a reduction to $75 \%$ IF did produce a decrement in concept identification performance. Procedural and task differences may account for this discrepancy between the two sets of results.

\section{References}

Bourne, L. E., Jr., \& Pendleton, R. B. Concept identification as a function of completeness and probability of information feedback. J. exp. Psychol., 1958, 56, 413-420.

Duncan, D. B. Multiple range and multiple F tests. Biometrics, $1955,11,1-42$.

Gelfand, S. Effects of prior associations and task complexity upon the identification of concepts. Psychol. Rep., 1958, 4, 567-574.

Namikas, G. Effect of verbal pretraining on performance in a concept identification task. (Unpublished doctoral dissertation, University of Wisconsin, Madison, 1962).

\section{Notes}

1. The research was presented in part at the 1966 meeting of the Midwestern Psychological Association.

2. The stimulus cards were made available through the courtesy of Dr. E. James Archer.

3. Analyses of variance were performed on errors-to-criterion and trials-to-criterion response measures. In the case of the second measure, a score of 128 was assigned when a $\mathrm{S}$ did not reach the 32 correct consecutive response criterion. The data from Experiment 1 were analyzed separately and in combination with the data from Experiment 2. The same significant effects were obtained irrespective of the response measure or whether the data from the experiments were combined or not. Consequently, only the results of the combined analysis for the error data are being presented. 